\title{
Precision control of tensile properties in fabric for computational fabrication
}

\author{
Ella Moore \\ Clemson University \\ ella@clemson.edu \\ Ioannis Karamouzas \\ Clemson University \\ ioannis@clemson.edu
}

\author{
Michael Porter \\ Clemson University \\ mmporte@clemson.edu \\ Victor Zordan \\ Clemson University \\ vbz@clemson.edu
}

\begin{abstract}
This paper presents an exploration of computer-controlled embroidery design and stitching with the goal of making purposeful, precision changes to material properties of a base elastic textile. Two techniques are proposed for adding stitches through designed microstructural cells and stitch-level planning. For the latter, a novel path planning algorithm is proposed to serialize stitches that is similar to a greedy solution for the travelling salesman problem with a set of domain-specific constraints that dictate edge cost. We show the efficacy of the concept through a set of simple design examples that undergo mechanical load testing and discuss the value of the technique in future applications using computational design.
\end{abstract}

\section{CCS CONCEPTS}

- Computing methodologies $\rightarrow$ Graphics systems and interfaces; Computer graphics;

\section{KEYWORDS}

computational fabrication, digital textile design

\section{ACM Reference Format:}

Ella Moore, Michael Porter, Ioannis Karamouzas, and Victor Zordan. 2018 Precision control of tensile properties in fabric for computational fabrication. In Proceedings of Symposium on Computational Fabrication (SCF'18). ACM, New York, NY, USA, 7 pages. https://doi.org/10.1145/3213512.3213514

\section{INTRODUCTION}

Modern textiles are advancing to include a wide spectrum of materials and weaves. However, the production of fabric artifacts that include multiple distinct properties most often requires the assembly (or layering) of materials through seaming and therefore can only be done at a coarse scale. An exception is knitting with distinct patterns which leads to limited control over the output material. We propose the combination of precision embroidery with computational design to produce a spectrum of material properties

Permission to make digital or hard copies of all or part of this work for personal or classroom use is granted without fee provided that copies are not made or distributed for profit or commercial advantage and that copies bear this notice and the full citation on the first page. Copyrights for components of this work owned by others than ACM must be honored. Abstracting with credit is permitted. To copy otherwise, or republish, to post on servers or to redistribute to lists, requires prior specific permission and/or a fee. Request permissions from permissions@acm.org.

SCF'18, fune 17-19 2018, Cambridge, MA USA

(C) 2018 Association for Computing Machinery.

ACM ISBN 978-1-4503-5854-5/18/06 . .\$15.00

https://doi.org/10.1145/3213512.3213514

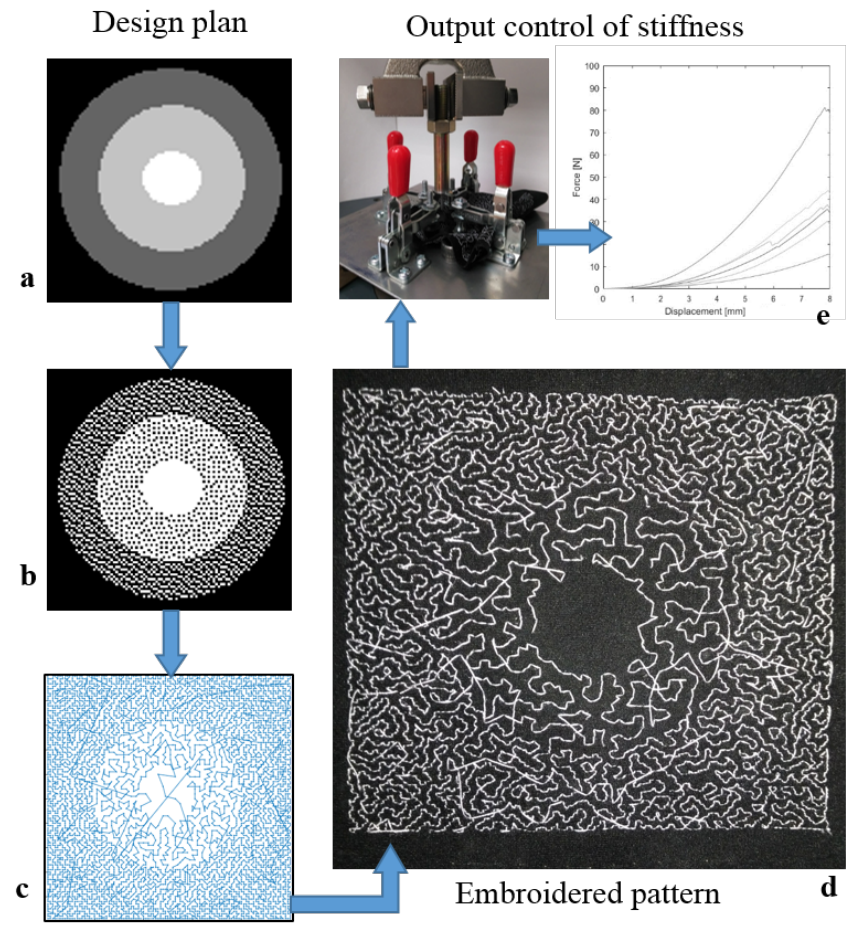

Figure 1: System layout. Starting from an initial design for a planned stitch density (a), we build a stitch layout (b) and serialized stitch path (c). This pattern is embroidered onto a base stretch material (d). Through mechanical testing we show the output effect on tensile properties (e).

within a single (unseamed) textile artifact. The result is a strong and precision controllable material that supports a wide spectrum of design options within many application fields from smart clothing to medical textiles.

At its core, embroidery changes the mechanical properties of fabrics. The strength, orientation, and distribution of stitches dictates the local properties in tension as well as (with enough stitching) in compression. Careful assembly of stitches with controlled directionality and order yield global mechanical properties that result from the aggregate effect of embroidered patterning and density. In this paper, we focus on increasing the local tensile strength of 
a 4-way-stretch elastane fabric to create tailored distributions of mechanical properties in the embroidered materials.

To this end, retooling of computer-driven embroidery in the production of materials with arbitrarily controlled stiffnesses raises an interesting set of technical challenges. For example, by its nature, an automatic embroiderer lays down a series of stitches in a connected fashion which requires planning to serialize stitches in alignment with control of the tensile properties, including tensile strength and stiffness. This paper repesents a step in the direction of such control through the following contributions: 1) the premiere exploration (to our knowledge) of embroidery as a means to change local material properties in a controllable and precise manner; 2 ) the proposal of two distinct techniques that aim to accomplish a stiffness design plan through metamaterial cells and at the embroidery stitch level; and 3) the introduction of a unique path planning approach that performs stitch serialization to accomplish precision stiffness control.

We showcase a set of examples for design using our techniques as well as results of puncture testing of physical samples to reveal the efficacy of our approach over material tensile strength.

\section{BACKGROUND}

Computational methods that control material properties have been the focus of research for several years [Bickel et al. 2010]. Recently, approaches have been proposed for 3D printing of various stiffness, including using microstructures [Panetta et al. 2015; Schumacher et al. 2015] and voronoi foams [Martínez et al. 2016] as well as socalled metasilicone [Zehnder et al. 2017] for injecting different types of materials to control stiffness. Similarly others have addressed the problem of building flexible rod meshes that automatically match input poses under boundary conditions [Pérez et al. 2015]. These are all part of a growing field in computational design that focuses on a variety of problems [Ion et al. 2016; Konaković et al. 2016; Skouras et al. 2014].

Within the field, there is a growing body of work that is looking at computational fabrication as it applies to the synthesis and incorporation of textiles and fabric objects. For example, the work of McCann and colleague addresses formalization of computational design as it applies to knitting by proposing a general knitting compiler [McCann et al. 2016]. The teams' recent 2018 paper offers an automatic approach to knitting from 3D meshes [Narayanan et al. 2018]. Other researchers have looked at a set of related problems, for example, origami with smart embroidery [Stoychev et al. 2017] that uses embroidering to generate material patterns to achieve actuation. Others include techniques to include fur/textile covering a 3D shape [Mahdavi-Amiri et al. 2015], 3D printing using felted fabric [Peng et al. 2016] and user-assisted 3D knitting [Igarashi et al. 2008a,b] as well as smart stretch sensors [Vogl et al. 2017] This work has also lead to a cluster of work on soft robotics.

Possibly the most related to our own work is that of Guseinov and his colleagues in their work in "CurveUps" [Guseinov et al 2017]. They combine 3D printing and stretch fabric to produce shaped 3D models from flat prints laid over pretensioned material. In comparison to the direction we propose, the material properties are not modified purposefully, but instead their goal is to use the stretch of the fabric to support the transformation into the three dimensional structure. Instead, we modify the fabric's mechanical properties in a deliberate fashion by stitching to control tensile response. Combining the embroidery with $3 \mathrm{D}$ printing on material opens interesting design possibilities for future work.

\section{METAMATERIAL DESIGN}

Computational embroidery holds promise for the design of tensile (and compression) properties for fabrics to a high degree of accuracy. However, the layout of the stitch plays an important role in the resulting properties.

\subsection{Stitch layout}

The design of stitch layout accounts for primary and secondary effects on the strength and deformation characteristics of embroidered fabrics. For example, threads stitched parallel to the primary direction of in-plane loading will lead to the greatest resistance, but will also tend to fail at very small displacements as a single stitch bears the entire load.

Although stiffening is reduced, a zigzag stitch can be used to avoid thread failure, producing a tailored stretch response that allows for some displacement while also increasing the local stiffness. Note, as the zigzag stretches, the base material also stretches and carries a portion of the load, unlike the straightline stitch.

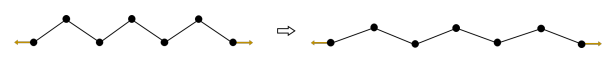

In two dimensions, further options become available (Figure 2), including the use of multiple stitch lines embroidered in parallel to increase unidirectional strength and stiffness (Figure 2, top). We call this the accordian stitch pattern and it can provide strong stiffening in one direction with relatively low stiffening in the orthogonal direction. Bi-directional stiffness results from consideration of multidirectional stitches across the material surface (Figure 2, middle). Here, both the choice of patterns as well as the stitch density (SD) yields a wide set of options that allow for smooth modification of the elastic base layer from little adjustment to highly stiff, inextensiblelike fabric properties (Figure 2,bottom).

\subsection{Stiffness control}

We explore two approaches to control the tensile properties of fabrics through purposeful stitch layout. Firstly, we follow an approach similar to that of the 3D print work by [Panetta et al. 2015; Schumacher et al. 2015], developing a series of variable-stiffness embroidery blocks (EB). The EB technique forms a meta-resolution that allows stacking of different EB elements to produce a desired change in (aggregate) stiffness. The second technique controls at the stitch-level (SL) through a density map and path planning (Section 4). Both techniques assume as input a stiffness design derived manually for the results shown. Automation of this design holds tremendous promise, but is outside of the scope of this paper.

The output of (either) stiffness control technique is an embroidery plan implemented in a separate pass. For EB, each block is a straightforward accordian pattern (shown at the top of the Figure 2) 

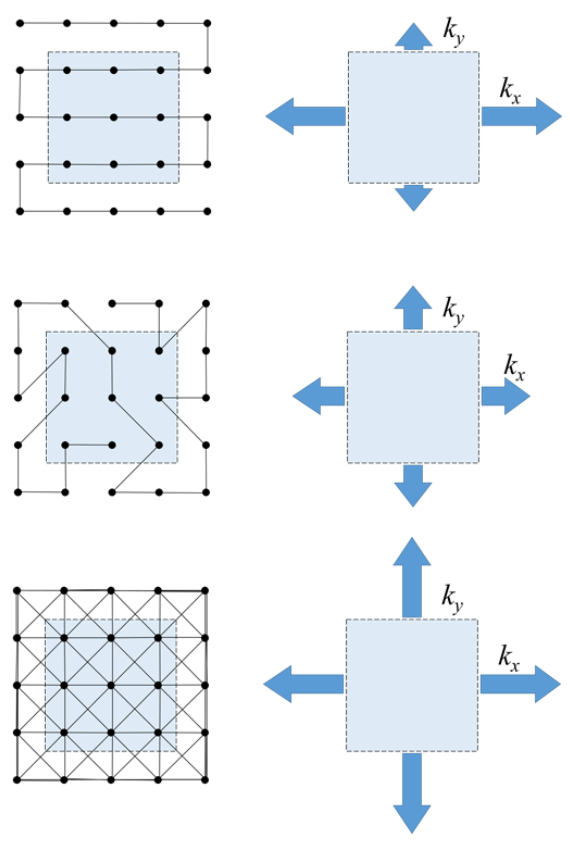

Figure 2: Sample 2D stitch layouts and their effect on stiffness of the material.

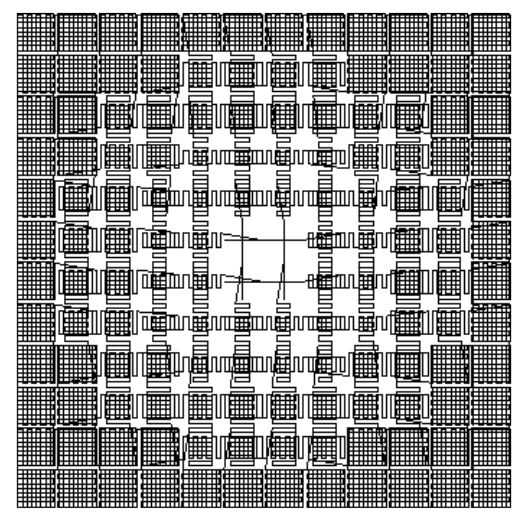

Figure 3: Accordian embroidery blocks (EB) assembled to produce a target with lower stiffness in its center. Three EB levels are allowed with simple rasterized stitch layout.

centered within the cell. The length of the pattern matches the size of the cell, and provides stitch connectivity from block to block. The width of the accordian pattern is set at three discrete values in our examples to create equidistant regions that produce successively more resistence orthogonal to the pattern, i.e. modulating stiffened and unmodified material along a series of similar blocks. For each $\mathrm{EB}$, two accordians appear, one horizontally and one vertically, running between the block's boundaries. We empirically selected a block element size of 10 stitches per side.
The EB technique is very easy to implement through simply coded stitch blocks of a set size, and the embroidery plan can be executed trivially through a raster-ordered layout in the final embroidery file. Figure 3 shows an example result. However, contrasting the size of the block relative to the size of the stitch, the EB approach yields a coarse discretization of the stiffness control based on the length of a single embroidery stitch, which is the hard lower limit of control for the embroidery hardware.

To achieve the goal of controlling stiffness at finer resolutions, ideally at the stitch level, we propose the SL approach which aggregates the general area of a set of stitches to control material strength through stitch density, SD. To solve such, we split the problem into two subproblems and solve each in turn. First, we identify the placement of stitches required based on the input stiffness design, and a known calibration. Second, in a path planning stage, we take the unordered stitches and produce a path that connects the stitches while upholding a number of constraints to complete the embroidery plan.

The placement for the SL stitches is performed through production of dither sampling. Specifically, starting from a desired stiffness design, we interpret a simple parametric normalized stiffness that holds a value from 0 , being no increases stiffness, to 1 or complete inextension. Under the assumption of uniform stiffening in both of the major (orthogonal) axes along the fabric surface, the sampling of the material is akin to producing a stipple pattern on a grayscale image. In this case, the stitch direction is irrelevant as long as the distribution of all stitches does not show a bias in directionality.
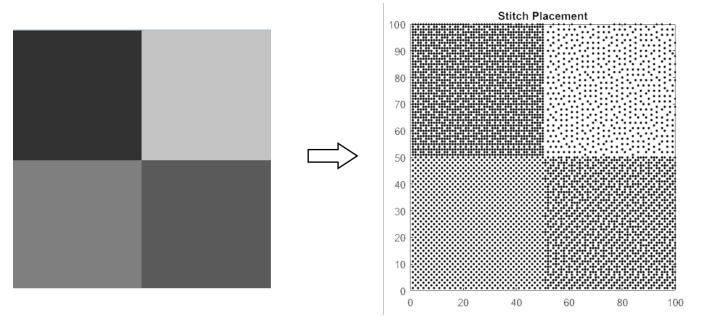

Figure 4: 2x2 sample densities and their sampling.

Adding jitter to the position of the samples leads to removal of undesired aliasing in the path planning stage described next.

\section{STITCH PLANNING}

The stitch planning problem is akin to coverage planning in robotics where a robot (e.g. a lawnmower) needs to touch all parts of a space or surface [LaValle 2006]. A number of solutions have been proposed for covering polygonal regions that rely on grid discretization and the use of boustrophedon paths (we refer the reader to [Choset 2001] for a related survey). When seeking for an optimal coverage, the problem is closely related to the traveling salesman problem (TSP), where the shortest tour that visits a given set points on the 2D plane must be found. As the TSP is NP-hard [Papadimitriou 1977], a number of approximation algorithms have been proposed, with the Christofides algorithm providing the best approximation ratio [Christofides 1976]. Various heuristics have 
also been devised that lead to good solutions such as the employment of a greedy nearest neighbor walk, and the k-opt heuristic that repeatedly swaps pairs of subtours to create a shorter tour [Rosenkrantz et al. 2009].

In our domain, we can treat the dither sampling of the stiffness plan as a set of points that must be visited. As compared to TSP, though, our application has a few unique characteristics. First, varying coverage density across regions is not explicitly described in our knowledge of the domain, but is key for our problem. Second, straight-line paths need to be avoided to promote equal load balancing across the surface of the fabric. Third, long stitches are not preferable (the longest stitch that our embroidery machine supports is $15 \mathrm{~mm}$ long). To account for these issues, we transform our planning problem to a variant of the traveling salesman problem.

Formally, let $G=(V, E)$ be the complete graph derived from the dither samples of the stiffness plan, where each vertex $\mathbf{v} \in V$ denotes a $2 \mathrm{D}$ point, and each edge $e=(\mathbf{u}, \mathbf{v}) \in E$ denotes a potential stitch between the vertices $\mathbf{u}$ and $\mathbf{v}$. Our goal is to find a path that visits all samples, while penalizing steps that are straight and avoiding making long stitches. To do so, we assign the following cost between two successive edges in the path:

$$
\cos t(\mathbf{u}, \mathbf{v}, \mathbf{w})=-\alpha^{-\|\mathbf{v}, \mathbf{w}\|} \beta^{-|\cos (\phi)|},
$$

where $\mathbf{u}, \mathbf{v}, \mathbf{w}$ are three successive stitch points, and $\phi$ is the angle between the edge $e=(\mathbf{u}, \mathbf{v})$ and its successor edge $e^{\prime}=(\mathbf{v}, \mathbf{w})$. The weighting constants $\alpha, \beta \geq 1$ control the importance of the two cost terms.

Given a stitch density map, we employ a heuristic solution to solve the aforementioned planning problem as summarized in $\mathrm{Al}$ gorithm 1 . We start from a random vertex in the dither graph and perform a greedy search where at each iteration the best unexplored vertex is selected based on (1). As a post-processing step, we refine our solution by employing a 2-opt heuristic approach that swaps one pair of edges for another pair with the same endpoints and shorter total length leading to a subsequence of the path to be reversed [Croes 1958].

In our implementation (cf. Algorithm 2), we only focus on improving edges that are longer than a user-defined threshold, and perform swaps in a lazy manner; we swap edges as soon as an improvement can be made rather than searching for the best possible swap. The 2-opt algorithm halts when there are no edges longer than the user-defined threshold. This secondary process allows us to remove the longest stitches in exchange for a modest amount of extra processing time.

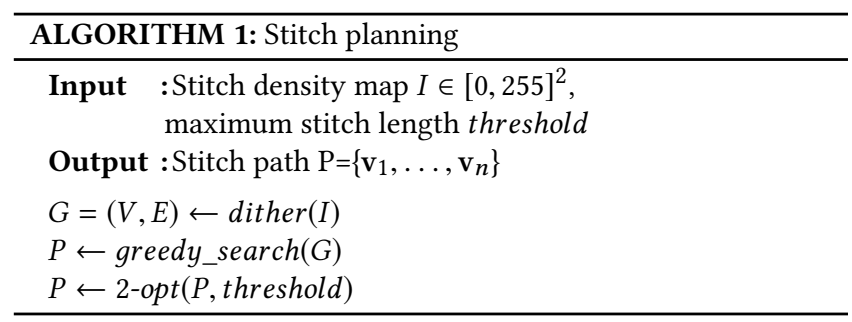

Figure 5 shows the two steps of our solution for a given stitch density map that was designed to enable cushion support for a water bottle base. After performing a greedy search, our approach

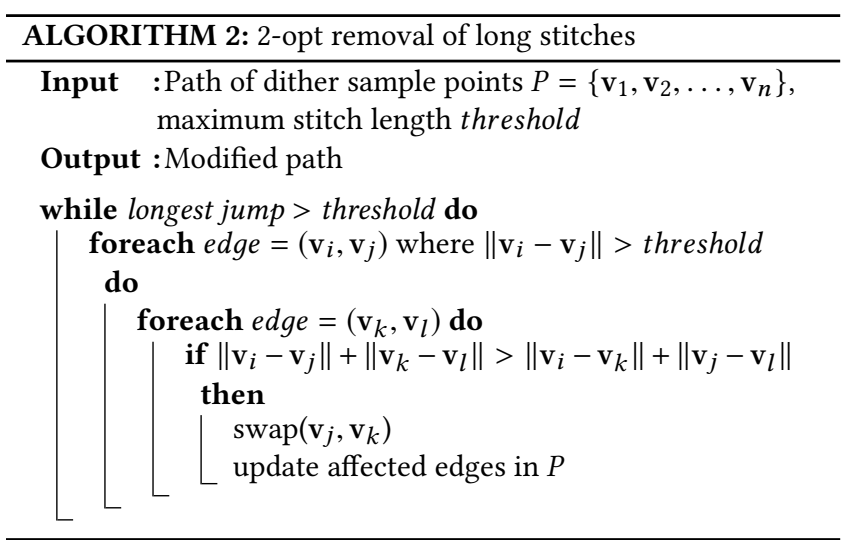

is able to return a path that follows the dithered stitch samples but has a number of stitches longer than a user-defined threshold of $10 \mathrm{~mm}$. By running the 2-opt post-processing step, a final stitch pattern is obtained with no long jumps.

It is worth mentioning that our approach can be run multiple times, where at each time a different greedy path is first obtained and then refined using the 2-opt heuristic. This allows us to better match the desired input stiffness map, and once more differentiates our problem from the traditional traveling salesman problem, as multiple points can be visited more than once.

\section{IMPLEMENTATION AND MECHANICAL TESTING}

We implement all embroidered results on a Brother SB7900E professional embroidery machine. All experiments use a medium weight 4-way elastane material with 50 weight poly embroidery thread. Black and white respectively are selected to clearly show the stitch pattern. Basic operating procedures (based on the machine instructions) were used for the machinery to tension both the material and thread. We found the use of a water-soluable film backing to hold the fabric in place lead to reduction in knotting. Average embroidery time for examples in this paper runs between 15-30mins depending on complexity.

Our stitching planning implementation currently runs in Matlab, and exports the computed path into a custom machine readable file that can be executed by the embroiderer. As our algorithm has a quadratic runtime complexity, its performance depends on the number of input dither samples. For example, the waterbottle cushion testcase shown in Figure 5 contains 6850 stitches. On an Intel i7-6550 single threaded CPU, it took 11.63 s to obtain a greedy path and 42.11 s to further refine this solution using the 2-opt heuristic. Qualitative results are shown under simple loads in Figure 7.

To quantify the tensile properties of various stitch patterns, we used a custom-built puncture device, with a $2.5 \mathrm{~mm}$ diameter puncture rod and $10.0 \mathrm{~mm}$ diameter test region, to apply radial, in-plane tensile stresses through selected circular regions of the embroidered fabrics. The puncture device was connected a universal materials testing machine with a $500 \mathrm{~N}$ load cell (Shimadzu AGS-X) and the fabric samples were tested at a displacement rate of $1.0 \mathrm{~mm} / \mathrm{sec}$ 

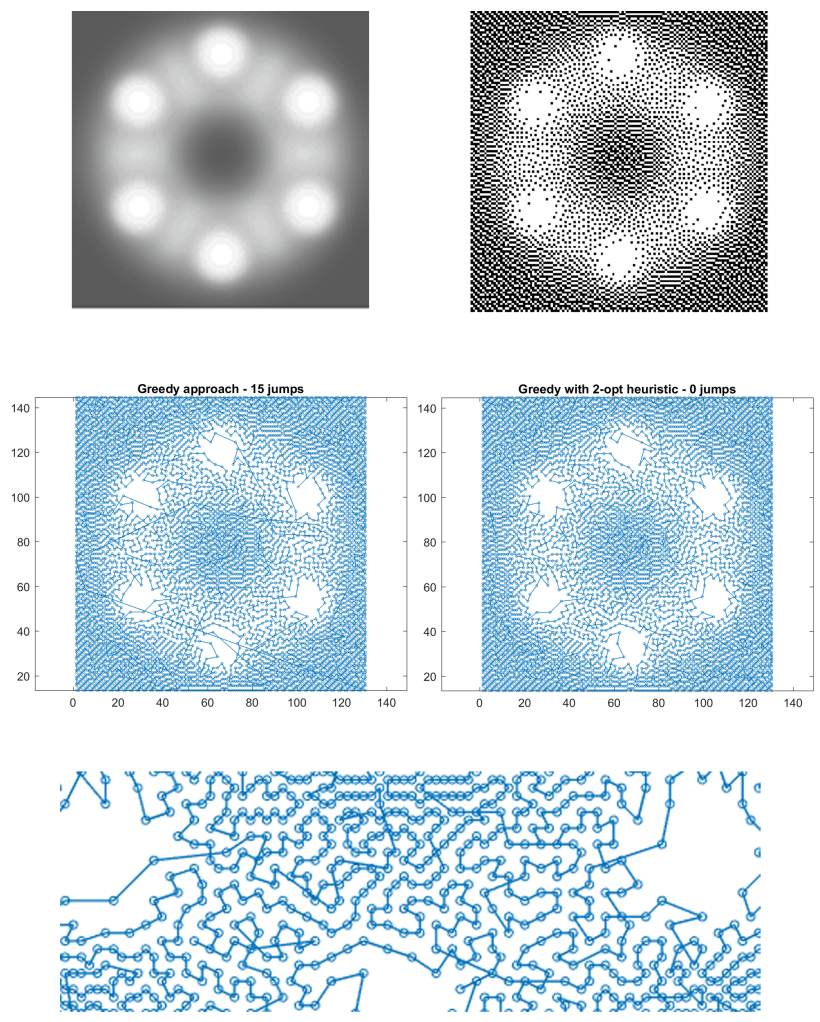

Figure 5: Design for cushion for waterbottle base. Top row: Stitch density map (left) and dithered stitch samples (right); Middle row: Output of greedy solution to TSP (left) Final stitch pattern after applying the 2-opt heuristic (right); Bottom row: Close up showing showing continuous stitch density change.

to a total displacement of $8.0 \mathrm{~mm}$, at which point the tests were interrupted.

Figure 8 shows an image of the test setup and the resulting forcedisplacement curves for six samples of uniform embroidery patterns and stitch densities. The stitch densities (SD) were approximated by equally adjusting the image thresholds of the different patterns, using ImageJ software (National Institutes of Health, Bethesda, MD). The top row of Figure 8 compares three samples of straightline stitches with increasing stitch densities, producing a notable increase in puncture strength and stiffness. In some samples (e.g. SD = 0.40), stitch failure was observed, indicating that the in-plane tensile stresses surpassed the strength of the embroidery thread - note this was more frequently observed in the medium-density straightline stitch patterns which (when sewn into orthogonal cross-stitches) bear the majority of the applied load.

The bottom row of Figure 8 compares three samples using the SL stitch layout. Note that comparable stitch density of these patterns in contrast to the straightline stitchs led to smaller, more gradual changes in puncture strength and stiffness (maximum load and slope of the force-displacement curves). This occurs because the stiffness of these patterns is more dependent on stitch layout than stitch density. That is, non-overlapping stitches tend to transfer transverse stresses through the base fabric. Hence, it is possible to tailor embroidery patterns such that stitches added to a base fabric can have either a minimal influence on aggregate stiffness (via non-overlapping, parallel stitches) or a maximal influence on aggregate stiffness (via overlapping cross-stitches).
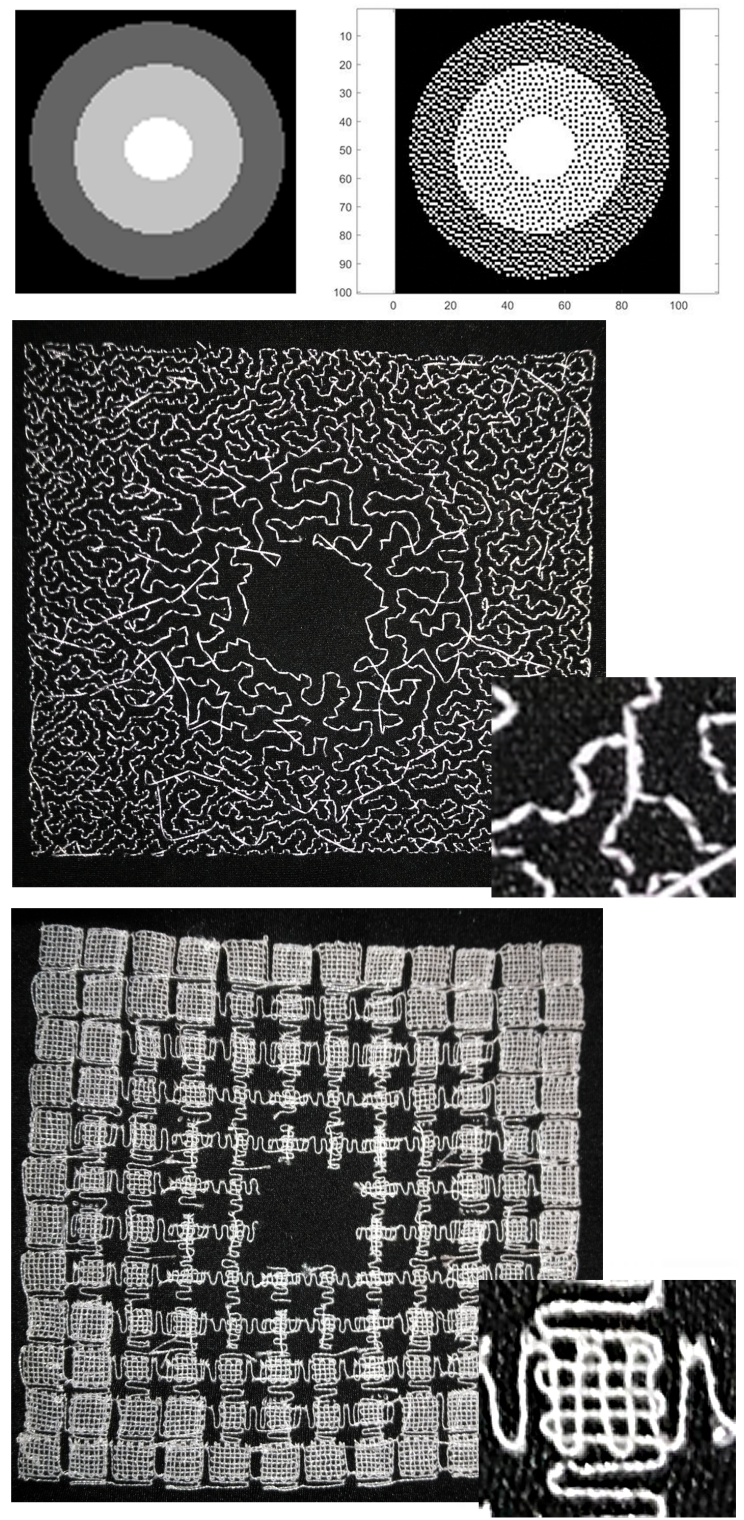

Figure 6: "Target" test pattern and dithered samples (top), the SD approach (middle) and EB approach (bottom) .

\section{DISCUSSION}

In conclusion, to our knowledge we present the first examples of embroidery being coupled with computational fabrication to yield textiles with varying tensile properties. We propose two approaches 

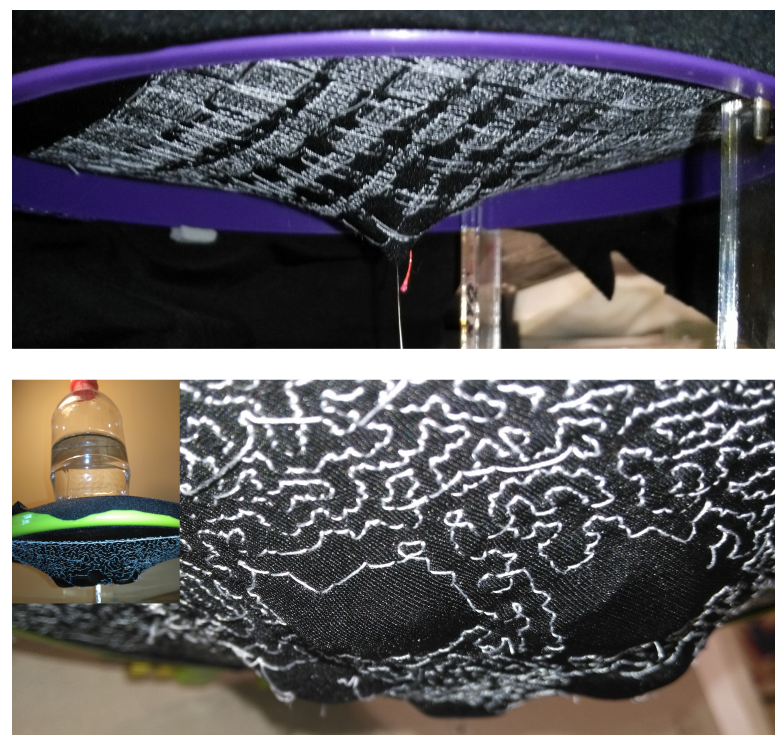

Figure 7: Results showing qualitative response of embroidered samples. Top: Target example with EB approach shows graded stiffness toward center of target. Bottom: Waterbottle cushion shows how stiffening contours and redistributes pressure under load.

to turn desired stiffness plans into realized samples that reveal quantitative tensile strength characteristics based on mechanical load testing.

While this paper is not focused on the practical applications of the proposed technique, there are a number of applications that would benefit from the described work. One example is akin to custom insoles that take a distinct pressure profile and build a unique insole to fit the individual. Here a similar product could be developed as a sling-style seat to allow a person that is wheelchair bound to relieve pressure that leads to bed sores. Smart clothing that shapes pressure profiles for blood circulation is another example for medical textiles (and may also be appropriate for performance apparel). Indeed, many medical textile applications focus on control for stiffness [Ng et al. 2017]. However, the goal for us is to show as a proof of concept the efficacy of the use of embroidery to control tensile properties.

There are a number of clear paths for future work. Foremost, following the computational fabrication research trends, computational design machinery with physical cloth simulation will help to automate and broaden the types of artifacts that can be produced. Combining the technique with 3D printing also broadens opportunities with some intriguing design elements where compression can be seamlessly added to a fabric.

The limitations of the current approach include the size and accuracy of the hardware which limit the resolution of the examples we can create. Further, relative to the EB solution, the SL stiffening lead to relatively small changes in the resulting tensile tests (as shown in Figure 8), largely due to reduced stitch density. We believe we can address this with the application of several layers of the embroidered patterns (among other solutions) and do not see this as a fundamental flaw of the approach. Regarding the stitch planning, our proposed algorithm acts locally, and as such we can make no guarantees that large jump stitches may be necessary. While a small number of jump stitches is tolerable (as they can be trimmed automatically by the embroiderer) we will look to improve the path serialization in future efforts.

Finally, an intriguing observation about future effort includes the development of non-uniform tensile properties. While we focus on uniform stiffening here, interpreting a full-color density map with $r g b$ values as the direction and magnitude of a vector field, we could produce an analogous stitch sampling with color representing the preferred direction of the stitch. While the path planning problem becomes more complicated (it is similar to coverage planning in the presence of an anisotropic guidance field), the result would be a controllable directed stiffening within a fabric which does not exist in today's textiles.

\section{ACKNOWLEDGMENTS}

This work was supported in part by the National Science Foundation under NSF Award CHS-172297 and the EPSCoR Program under NSF Award OIA-1655740

\section{REFERENCES}

Bernd Bickel, Moritz Bächer, Miguel A. Otaduy, Hyunho Richard Lee, Hanspeter Pfister, Markus Gross, and Wojciech Matusik. 2010. Design and Fabrication of Materials with Desired Deformation Behavior. ACM Trans. Graph. 29, 4, Article 63 (July 2010), 10 pages. DOI : http://dx.doi.org/10.1145/1778765.1778800

Howie Choset. 2001. Coverage for robotics-A survey of recent results. Annals of mathematics and artificial intelligence 31, 1-4 (2001), 113-126.

Nicos Christofides. 1976. Worst-case analysis of a new heuristic for the travelling salesman problem. Technical Report. Carnegie-Mellon Univ Pittsburgh Pa Management Sciences Research Group.

Georges A Croes. 1958. A method for solving traveling-salesman problems. Operations research 6, 6 (1958), 791-812.

Ruslan Guseinov, Eder Miguel, and Bernd Bickel. 2017. CurveUps: Shaping Objects from Flat Plates with Tension-actuated Curvature. ACM Trans. Graph. 36, 4, Article 64 (July 2017), 12 pages. DOI : http://dx.doi.org/10.1145/3072959.3073709

Yuki Igarashi, Takeo Igarashi, and Hiromasa Suzuki. 2008a. Knitting a 3D model. In Computer Graphics Forum, Vol. 27. Wiley Online Library, 1737-1743.

Yuki Igarashi, Takeo Igarashi, and Hiromasa Suzuki. 2008b. Knitty: 3D Modeling of Knitted Animals with a Production Assistant Interface.. In Eurographics (Short Papers). Citeseer, 17-20.

Alexandra Ion, Johannes Frohnhofen, Ludwig Wall, Robert Kovacs, Mirela Alistar, Jack Lindsay, Pedro Lopes, Hsiang-Ting Chen, and Patrick Baudisch. 2016. Metamaterial Mechanisms. In Proceedings of the 29th Annual Symposium on User Interface Software and Technology (UIST '16). ACM, New York, NY, USA, 529-539. DOI : http://dx.doi. org/10.1145/2984511.2984540

Mina Konaković, Keenan Crane, Bailin Deng, Sofien Bouaziz, Daniel Piker, and Mark Pauly. 2016. Beyond Developable: Computational Design and Fabrication with Auxetic Materials. ACM Trans. Graph. 35, 4, Article 89 (July 2016), 11 pages. DOI: http://dx.doi.org/10.1145/2897824.2925944

Steven M LaValle. 2006. Planning algorithms. Cambridge university press.

Ali Mahdavi-Amiri, Philip Whittingham, and Faramarz Samavati. 2015. Cover-it: An Interactive System for Covering 3D Prints. In Proceedings of the 41st Graphics Interface Conference (GI '15). Canadian Information Processing Society, Toronto, Ont., Canada, Canada, 73-80. http://dl.acm.org/citation.cfm?id=2788890.2788905

Jonàs Martínez, Jérémie Dumas, and Sylvain Lefebvre. 2016. Procedural Voronoi Foams for Additive Manufacturing. ACM Trans. Graph. 35, 4, Article 44 (July 2016), 12 pages. DOI : http://dx.doi.org/10.1145/2897824.2925922

James McCann, Lea Albaugh, Vidya Narayanan, April Grow, Wojciech Matusik, Jennifer Mankoff, and Jessica Hodgins. 2016. A Compiler for 3D Machine Knitting. ACM Trans. Graph. 35, 4, Article 49 (July 2016), 11 pages. DOI : http://dx.doi.org/10.1145/ 2897824.2925940

Vidya Narayanan, Lea Albaugh, Jessica Hodgins, Stelian Coros, and James McCann. 2018. Automatic Machine Knitting of 3D Meshes. ACM Trans. Graph. 37, 4 (July 

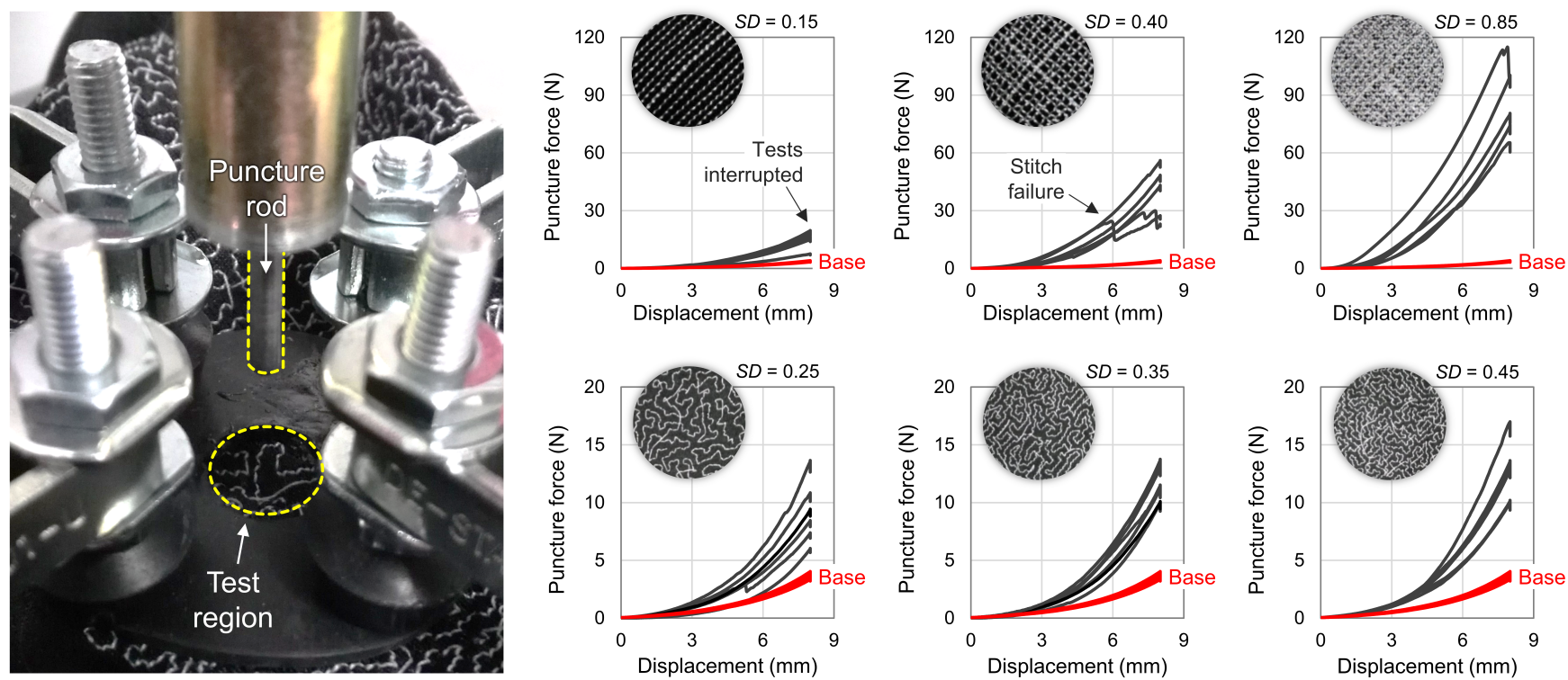

Figure 8: Left: Experiemental Set-up for Puncture Test. Righti: Results from puncture tests for various samples. Each sample is tested multiple times, results in black compared to base material in red. Top row show example straightline stitches, bottom row show results from SL approach.

2018).

Joanna L Ng, Ciara E Collins, and Melissa L Knothe Tate. 2017. Engineering mechanical gradients in next generation biomaterials-Lessons learned from medical textile design. Acta biomaterialia 56 (2017), 14-24.

Julian Panetta, Qingnan Zhou, Luigi Malomo, Nico Pietroni, Paolo Cignoni, and Denis Zorin. 2015. Elastic Textures for Additive Fabrication. ACM Trans. Graph. 34, 4, Article 135 (July 2015), 12 pages. DOI : http://dx.doi.org/10.1145/2766937

Christos H Papadimitriou. 1977. The Euclidean travelling salesman problem is NPcomplete. Theoretical computer science 4, 3 (1977), 237-244.

Huaishu Peng, Scott Hudson, Jennifer Mankoff, and James McCann. 2016. Soft printing with fabric. XRDS: Crossroads, The ACM Magazine for Students 22, 3 (2016), 50-53.

Jesús Pérez, Bernhard Thomaszewski, Stelian Coros, Bernd Bickel, José A. Canabal, Robert Sumner, and Miguel A. Otaduy. 2015. Design and Fabrication of Flexible Rod Meshes. ACM Trans. Graph. 34, 4, Article 138 (July 2015), 12 pages. DOI: http://dx.doi.org/10.1145/2766998

Daniel J Rosenkrantz, Richard E Stearns, and Philip M Lewis. 2009. An analysis of several heuristics for the traveling salesman problem. In Fundamental Problems in Computing. Springer, 45-69.

Christian Schumacher, Bernd Bickel, Jan Rys, Steve Marschner, Chiara Daraio, and Markus Gross. 2015. Microstructures to Control Elasticity in 3D Printing. ACM Trans. Graph. 34, 4, Article 136 (July 2015), 13 pages. DOI : http://dx.doi.org/10.1145/ 2766926

Mélina Skouras, Bernhard Thomaszewski, Peter Kaufmann, Akash Garg, Bernd Bickel, Eitan Grinspun, and Markus Gross. 2014. Designing Inflatable Structures. ACM Trans. Graph. 33, 4, Article 63 (July 2014), 10 pages. DOI : http://dx.doi.org/10.1145/ 2601097.2601166

Georgi Stoychev, Mir Jalil Razavi, Xianqiao Wang, and Leonid Ionov. 2017. 4D Origami by Smart Embroidery. Macromolecular rapid communications 38, 18 (2017).

Anita Vogl, Patrick Parzer, Teo Babic, Joanne Leong, Alex Olwal, and Michael Haller. 2017. StretchEBand: Enabling Fabric-based Interactions Through Rapid Fabrication of Textile Stretch Sensors. In Proceedings of the 2017 CHI Conference on Human Factors in Computing Systems (CHI '17). ACM, New York, NY, USA, 2617-2627. DOI : http://dx.doi.org/10.1145/3025453.3025938

Jonas Zehnder, Espen Knoop, Moritz Bächer, and Bernhard Thomaszewski. 2017. Metasilicone: design and fabrication of composite silicone with desired mechanical properties. ACM Transactions on Graphics (TOG) 36, 6 (2017), 240. 